\title{
Small Neutrino Masses From Structural cancellation In Left-Right Symmetric Model
}

\author{
M.J.Luo* \\ Department of Modern Physics, \\ University of Science and Technology of China, \\ Hefei, Anhui 230026, China \\ Q.Y.Liu \\ Department of Modern Physics, \\ University of Science and Technology of China, \\ Hefei, Anhui 230026, China
}

\begin{abstract}
The Type I, II and hybrid (I+II) seesaw mechanism, which explain why neutrinos are especially light, are consequences of the left-right symmetric model (LRSM). They can be classified by the ranges of parameters of LRSM. We show that a nearly cancellation in general Type-(I+II) seesaw is more natural than other types of seesaw in the LRSM if we consider their stability against radiative correction. In this scenario the small neutrino masses are due to the structure cancellation, and the masses of the right handed neutrino can be of order of $\mathrm{O}(10) \mathrm{TeV}$. The realistic model for non-zero neutrino masses, charged lepton masses and lepton tribimaximal mixing can be implemented by embedding $A_{4}$ flavor symmetry in the model with perturbations to the textures.
\end{abstract}

*E-mail: mjluo@mail.ustc.edu.cn 


\section{INTRODUCTION}

The fact that neutrinos have very small masses has been established by a number of neutrino oscillation experiments [1] in the past decade, which is an important evident to go beyond the Standard Model. In order to generate very tiny neutrino masses, the very popular explanation is the seesaw mechanism [2].

In the so-called Type-I seesaw [2], extra very heavy Majorana right handed neutrinos $(\mathrm{RHN})$ are introduced. When integrating them out, the neutrino mass is approximately $m_{\nu} \sim m_{D}^{2} / m_{R}$, so we assume that the $m_{D}$ (the neutrino Dirac mass) is of the electroweak scale, i.e. $m_{D} \sim O\left(10^{2} \mathrm{GeV}\right)$, we need the RHN mass $m_{R} \sim O\left(10^{16} \mathrm{GeV}\right)$ which is hopeless to reach to direct test this mechanism. In the Type-II seesaw (triplet seesaw) [3], a heavy Higgs triplet $\Delta$ is introduced to play the similar role of heavy right handed neutrino to suppress the neutrino masses, we have $m_{\nu} \sim v^{2} / m_{\Delta}$, where $m_{\Delta} \sim O\left(10^{16} \mathrm{GeV}\right)$ is the mass of the Higgs triplet. In a general hybrid Type-(I+II) seesaw model, both terms make contributions to the neutrino masses. The crucial feature of such mechanisms are introducing heavy particles to suppress the neutrino masses, but the smallness of neutrino mass needs them to be too heavy to have any signals in future colliders.

Possible compromise between the impossible collider signals of such heavy particles and the smallness of neutrino masses is discussed in recent literatures in the framework of hybrid Type-(I+II) seesaw [4], where the small neutrino masses is from the structural cancellation, while suppression plays no role. In such scenario, the introduced heavy particles can be light enough to be direct produced in future colliders without violating the current bounds [5], so the possibilities have not been ruled out by experimental limits so far.

These types of seesaw are consequences of the left-right symmetric model (LRSM) [6], which is a possible extension of SM. In the model, unlike the SM that has only $S U(2)$ left handed chiral matter, the right handed sector under Non-Abelian $S U(2)$ representation are also introduced and correlated to the left handed sector. The LRSM not only leads to the seesaw mechanism but also provides explanation of the observed maximal $\mathrm{P}$ and $\mathrm{C}$ violation at low energy weak interaction, and is therefore likely in certain sense to be the final theory. The type of seesaw deduced from LRSM is determined by the space of parameters of the model. If we consider the stability of the parameters under the radiative correction, a model is "natural" if it is stable against the quantum correction, so fine-tuning for parameters is not needed. Before the mechanism can be tested directly in experiments, the naturalness is inevitable an important criteria for our model buildings.

In this paper, we will deduce the three types of seesaw in the LRSM and classify them by the ranges that the parameters locate. The 1-loop quantum correction of the parameter is evaluated and we find that the small neutrino mass from nearly cancellation in Type$(\mathrm{I}+\mathrm{II})$ is more "natural" than other types of seesaw in the limit of small couplings in Higgs potential. So unlike the literature [4] where the cancellation relation is imposed by hands, the structure cancellation in LRSM is a natural result of the model. Therefore, in this scenario, the RHN can be light and be of order of $\mathrm{O}(10) \mathrm{TeV}$. Finally, non-zero neutrino masses, charged lepton masses and tribimaximal mixing [7] are generated by perturbations and embedding an extra $A_{4}[8]$ flavor symmetry into the model. 


\section{THE MODEL}

\section{A. The Left-Right Symmetric Model}

The left-right symmetric model is based on the extended gauge group $G_{L R}=S U(2)_{L} \otimes$ $S U(2)_{R} \otimes U(1)_{B-L}$, in which a Higgs bi-doublet $\Phi$ and left (right) Higgs triplet $\Delta_{L(R)}$ are introduced and with the representation assignments

$$
\Phi \sim(2,2,0), \Delta_{L} \sim(3,1,2), \Delta_{R} \sim(1,3,2)
$$

Under a discrete left-right symmetry, $l_{L} \leftrightarrow l_{R}^{c}, \Delta_{L} \leftrightarrow \Delta_{R}$ and $\Phi \leftrightarrow \Phi^{T}$, the invariant Lagrangian of the Yukawa interaction term is

$$
-\mathcal{L}=y \bar{l}_{L} \Phi l_{R}+\tilde{y} \bar{l}_{L} \tilde{\Phi} l_{R}+\frac{1}{2} f\left[\bar{l}_{L} i \tau_{2} \Delta_{L} l_{L}^{c}+\bar{l}_{R}^{c} i \tau_{2} \Delta_{R} l_{R}\right]+\text { h.c. }
$$

where $l_{L(R)}=\left(\begin{array}{ll}\nu_{L(R)} & e_{L(R)}\end{array}\right)^{T}$ is the lepton doublet, $\tilde{\Phi}=\tau_{2} \Phi^{*} \tau_{2}, l_{L(R)}^{c} \equiv C{\overline{l_{L(R)}}}^{T}$ with $C$ being the charge-conjugation matrix. At first stage, the symmetry spontaneously broken into $S U(2)_{L} \times U(1)_{Y}$ by a non-zero vacuum expectation value (VEV) of $\Delta_{R}$, leading to a heavy Majorana mass for right handed neutrinos. The second stage, the $\Phi$ develops VEV, breaking the symmetry to relic $U(1)_{\mathrm{em}}$. The developed non-zero VEV consistent with $U(1)_{\mathrm{em}}$ electromagnetic invariance are

$$
\left\langle\Delta_{L}\right\rangle=\left(\begin{array}{cc}
0 & 0 \\
v_{L} & 0
\end{array}\right), \quad\left\langle\Delta_{R}\right\rangle=\left(\begin{array}{cc}
0 & 0 \\
v_{R} & 0
\end{array}\right), \quad\langle\Phi\rangle=\left(\begin{array}{cc}
v & 0 \\
0 & v^{\prime}
\end{array}\right) .
$$

The measurement of the $\rho$ parameter [9] constrains the tree-level contribution of the Higgs triplet, $v_{L} \lesssim 1 G e V$, which is much smaller than the electroweak scale $v \simeq 174 G e V$, and we will work in the approximation $v^{\prime} \ll v$. Integrating out the heavy fields the effective mass of neutrino can be written as the general Type-(I+II) seesaw formula

$$
M_{\nu} \simeq M_{L}-M_{D} M_{R}^{-1} M_{D}^{T}=v_{L} f-\frac{v^{2}}{v_{R}} y f^{-1} y^{T}
$$

The dominant contribution from the first or second term determines the type of seesaw. In the model the charged lepton and Dirac neutrino mass matrix are simply obtained as $M_{e}=\tilde{y} v I$ and $M_{D}=y v I$ ( $I$ is the identity matrix), which we will discuss and implement by introducing flavor symmetry in section IV.

\section{B. Higgs Potential}

Our aim here is to show the relations between the VEVs of the Higgs fields in LRSM, for this purpose, let us write the Higgs potential involving $\Phi$ and $\Delta_{L(R)}$. The most general renormalizable Higgs fields potential has the quadratic and quartic coupling terms and can not have any trilinear terms. So consistent with the transformation properties as Eq(1) and discrete left-right symmetry, the Higgs potential can be written as [10] 


$$
\begin{aligned}
V\left(\Phi, \Delta_{L}, \Delta_{R}\right) & =-\mu_{i j}^{2} \operatorname{tr}\left[\Phi_{i}^{\dagger} \Phi_{j}\right]+\lambda_{i j k l} \operatorname{tr}\left[\Phi_{i}^{\dagger} \Phi_{j}\right] \operatorname{tr}\left[\Phi_{k}^{\dagger} \Phi_{l}\right]+\lambda_{i j k l}^{\prime} \operatorname{tr}\left[\Phi_{i}^{\dagger} \Phi_{j} \Phi_{k}^{\dagger} \Phi_{l}\right] \\
& -\mu^{2} \operatorname{tr}\left[\Delta_{L}^{\dagger} \Delta_{L}+\Delta_{R}^{\dagger} \Delta_{R}\right]+\rho_{1}\left[\left(\operatorname{tr}\left[\Delta_{L}^{\dagger} \Delta_{L}\right]\right)^{2}+\left(\operatorname{tr}\left[\Delta_{R}^{\dagger} \Delta_{R}\right]\right)^{2}\right] \\
& +\rho_{2}\left(\operatorname{tr}\left[\Delta_{L}^{\dagger} \Delta_{L} \Delta_{L}^{\dagger} \Delta_{L}\right]+\operatorname{tr}\left[\Delta_{R}^{\dagger} \Delta_{R} \Delta_{R}^{\dagger} \Delta_{R}\right]\right)+\rho_{3} \operatorname{tr}\left[\Delta_{L}^{\dagger} \Delta_{L} \Delta_{R}^{\dagger} \Delta_{R}\right] \\
& +\alpha_{i j} \operatorname{tr}\left[\Phi_{i}^{\dagger} \Phi_{j}\right]\left(\operatorname{tr}\left[\Delta_{L}^{\dagger} \Delta_{L}\right]+\operatorname{tr}\left[\Delta_{R}^{\dagger} \Delta_{R}\right]\right) \\
& +\beta_{i j}\left(\operatorname{tr}\left[\Delta_{L}^{\dagger} \Delta_{L} \Phi_{i} \Phi_{j}^{\dagger}\right]+\operatorname{tr}\left[\Delta_{R}^{\dagger} \Delta_{R} \Phi_{i} \Phi_{j}^{\dagger}\right]\right) \\
& +\gamma_{i j}\left(\operatorname{tr}\left[\Delta_{L}^{\dagger} \Phi_{i} \Delta_{R} \Phi_{j}^{\dagger}\right]+\text { h.c. }\right),
\end{aligned}
$$

where the sums over $i, j, k$ and $l$ run from 1 to 2 , with $\Phi_{1}=\Phi$ and $\Phi_{2}=\tilde{\Phi}$. To recover the left-right symmetry and hermicity condition, the couplings satisfy the constraints,

$$
\begin{array}{r}
\mu_{i j}=\mu_{j i}, \lambda_{1212}=\lambda_{2121}, \lambda_{i i j k}=\lambda_{i i k j}, \\
\lambda_{i j k k}=\lambda_{j i k k}, \lambda_{i j k l}^{\prime}=\lambda_{l i j k}^{\prime}=\lambda_{k l i j}^{\prime}=\lambda_{j k l i}^{\prime}, \\
\alpha_{i j}=\alpha_{j i}, \beta_{i j}=\beta_{j i}, \gamma_{i j}=\gamma_{j i} .
\end{array}
$$

After the Higgs fields develop their VEV, we obtain

$$
V=-\mu^{2}\left(v_{L}^{2}+v_{R}^{2}\right)+\frac{\rho}{4}\left(v_{L}^{4}+v_{R}^{4}\right)+\frac{\rho^{\prime}}{2} v_{L}^{2} v_{R}^{2}+\frac{\alpha}{2}\left(v_{L}^{2}+v_{R}^{2}\right) v^{2}+\gamma v_{L} v_{R} v^{2},
$$

where the approximation $v^{\prime} \ll v$ is used, and the coefficients are

$$
\begin{gathered}
\gamma=2 \gamma_{12}, \\
\alpha=2\left(\alpha_{11}+\alpha_{22}+\beta_{11}\right), \\
\rho=4\left(\rho_{1}+\rho_{2}\right), \\
\rho^{\prime}=2 \rho_{3} .
\end{gathered}
$$

From the minimizing condition $\frac{\partial V}{\partial v_{L}}=\frac{\partial V}{\partial v_{R}}=0$, if $v_{L} \neq v_{R}$, we get the relations for VEV of Higgs fields,

$$
v_{L} v_{R}=\frac{\gamma}{\kappa} v^{2}
$$

where $\kappa=\rho-\rho^{\prime}$. The mass $m_{L}, m_{R}$ and $m_{D}$ will be of order of $v_{L}, v_{R}$ and $v$, respectively. In the next section, we will classify the types of seesaw mechanism generated from LRSM by the values of the ratio of Higgs particle self-couplings $\frac{\gamma}{\kappa}$.

\section{THE SEESAW TYPE AND STABILITY}

We now discuss their contributions to the neutrino masses. Substituting the relation $\mathrm{Eq}(9)$ into the general Type-(I+II) seesaw formula $\mathrm{Eq}(4)$, we get

$$
m_{\nu}=\left(f\left(\frac{\gamma}{\kappa}\right)-\frac{y^{2}}{f}\right) \frac{v^{2}}{v_{R}} .
$$

According to the formula, following classification can be given. 
1) Type-I seesaw: $f\left(\frac{\gamma}{\kappa}\right) \ll \frac{y^{2}}{f}$. It responds to the case of $m_{\nu} \simeq-\frac{y^{2}}{f} \frac{v^{2}}{v_{R}}=-m_{D} m_{R}^{-1} m_{D}^{T}$ dominant, the small neutrino mass is from the suppression of heavy $v_{R}$.

2) Type-II seesaw: $f\left(\frac{\gamma}{\kappa}\right) \gg \frac{y^{2}}{f}$. The term $m_{\nu} \simeq v_{L} f=m_{L}$ dominant, while $m_{D} m_{R}^{-1} m_{D}^{T}$ can be relatively neglected, i.e. the small neutrino mass is due to the smallness of $v_{L}$.

3) Nearly cancellation Type-(I+II) seesaw: $f\left(\frac{\gamma}{\kappa}\right) \simeq \frac{y^{2}}{f}$. The term $m_{L}$ and $m_{D} m_{R}^{-1} m_{D}^{T}$ are comparable in magnitude and will nearly cancel their contributions to get small neutrino mass, we will see that this scenario is radiative stable.

However it is classical value at tree level, here we want to explore the behavior of the $\frac{\gamma}{\kappa}$ defined at the scale $\mu_{0}$ under the radiative correction. The correction of $\gamma$ and $\kappa$ come from the 1-loop correction of the quartic coupling of operators $\Delta_{L} \Phi \Delta_{R} \Phi$ and $\Delta \Delta \Delta \Delta$, respectively. The renormalization group equation for $\gamma$ and $\kappa$ take the forms

$$
\begin{array}{r}
\mu \frac{d \gamma}{d \mu}=\frac{1}{16 \pi^{2}}\left[\left(a_{1} \alpha^{2}+a_{2} \beta^{2}+a_{3} \gamma^{2}\right)+\left(b_{1} \alpha+b_{2} \beta+b_{3} \gamma\right) y^{2}\right. \\
\left.+\left(c_{1} \alpha+c_{2} \beta+c_{3} \gamma\right) f^{2}+\left(d_{1} \alpha+d_{2} \beta+d_{3} \gamma\right) g^{2}+e_{1} g^{4}+e_{2} f^{2} y^{2}\right] \\
\mu \frac{d \kappa}{d \mu}=\frac{1}{16 \pi^{2}}\left[\left(a_{1}^{\prime} \rho_{1}^{2}+a_{2}^{\prime} \rho_{2}^{2}+a_{3}^{\prime} \rho_{3}^{2}\right)+\left(b_{1}^{\prime} \rho_{1}+b_{2}^{\prime} \rho_{2}+b_{3}^{\prime} \rho_{3}\right) f^{2}\right. \\
\left.+\left(c_{1}^{\prime} \rho_{1}+c_{2}^{\prime} \rho_{2}+c_{3}^{\prime} \rho_{3}\right) g^{2}+d_{1}^{\prime} g^{4}+d_{2}^{\prime} f^{4}\right]
\end{array}
$$

in which the coefficients $a, b, c, d, e$ are constants of order $O(1)$ that are determined by computing the corresponding 1-loop Feynman diagrams. $\alpha_{i j}, \beta_{i j}, \gamma_{i j}, \rho_{i}$ are coupling constants in Higgs potential $\mathrm{Eq}(5)$ and $g$ the gauge coupling.

The Yukawa couplings $f$ and $y$ are of order $O(1)$, but the typical coupling constants in Higgs potential and the gauge coupling are generally assumed to be much smaller than that. In fact, for large couplings, higher order or non-perturbative correction should be considered and we will not discuss them here. So we assume in this paper that in $\operatorname{Eq}(11)$ they can be approximately dropped, while only the loops that attribute to Yukawa couplings $f, y$ play dominant role. We estimate the magnitude of the 1-loop corrections at scale $\mu$ to be

$$
\begin{gathered}
\delta \gamma \simeq \frac{-n_{f} f^{2} y^{2}}{16 \pi^{2}} \ln \left(\frac{\mu}{\mu_{0}}\right), \\
\delta \kappa \simeq \frac{-n_{f} f^{4}}{16 \pi^{2}} \ln \left(\frac{\mu}{\mu_{0}}\right),
\end{gathered}
$$

where $n_{f}$ is the number of fermion species. The parameter $\frac{\gamma}{\kappa}$ is stable only when

$$
0=\delta\left(\frac{\gamma}{\kappa}\right)=\frac{(\delta \gamma) \kappa-\gamma(\delta \kappa)}{\kappa^{2}}
$$

so we get the relation

$$
\frac{\gamma}{\kappa} \simeq \frac{y^{2}}{f^{2}}
$$

which is consistent with the nearly cancellation type $f\left(\frac{\gamma}{\kappa}\right) \simeq \frac{y^{2}}{f}$. In other words, if $m_{\nu} \simeq 0$ in $\mathrm{Eq}(10)$ arises from the cancellation between $f\left(\frac{\gamma}{\kappa}\right)$ and $\frac{y^{2}}{f}$, because of $\mathrm{Eq}(13)$ it will lead to 
the stable value of $\frac{\gamma}{\kappa}$ that suppresses its radiative correction. Therefore, it is indicated that the scenario of nearly cancellation Type-(I+II) seesaw is more natural than other types of seesaw when we consider the factor of their stability. The neutrino mass is vanished when the cancellation relation $\frac{\gamma}{\kappa}=\frac{y^{2}}{f^{2}}$ is exactly hold as is shown in $\operatorname{Eq}(10)$.

The vanishing $m_{\nu}$ can also eliminate another unnaturalness that the texture of $f$ is not uniquely determined in LRSM [11], e.g. if $f$ is allowed, then so is $\hat{f}=\frac{m_{\nu}}{v_{L}}-f$. We can see that when $m_{\nu}=0, f$ is uniquely determined up to an unimportant phase or sign.

In this case, the $v_{R}$ does not need to play the role of suppressing the neutrino mass, the RHN mass can be scale of $O(10) \mathrm{TeV}$ by the constraints of $v_{L} \lesssim 1 \mathrm{GeV}$. This possibility that $v_{R}$ can be reachable $\mathrm{TeV}$ scale has not been ruled out by current bounds of experiments [5].

\section{NON-ZERO NEUTRINO MASSES AND TRIBIMAXIMAL MIXING}

The textures of Yukawa matrices discussed above are simple, in which the Dirac neutrino masses and the ones coming from the left(right) Higgs triplet are degenerate,

$$
\begin{array}{r}
M_{D}=y v I, \\
M_{L(R)}=f v_{L(R)} I .
\end{array}
$$

The neutrino is massless when the cancellation relation is hold. However, the masses of neutrino are not trivially vanished. So we will discuss a deviation from this scenario by perturbations and introducing flavors symmetry to get a more realistic model.

We embed the extra $A_{4}$ symmetry [8] into LRSM by the assignments

$$
l_{L(R)}, l_{L(R)}^{c} \sim \underline{\mathbf{3}}, \Phi \sim \underline{\mathbf{1}}, \Delta_{L(R)} \sim \underline{\mathbf{1}}
$$

where, in $A_{4}$ group, $\underline{\mathbf{3}}$ stands for the real three-dimensional irreducible representation and $\underline{\mathbf{1}}$ for the trivial one in the three inequivalent one-dimensional representations $\underline{\mathbf{1}}, \underline{\mathbf{1}}$, $\underline{\mathbf{1}}^{\prime}$. So the invariant Yukawa Lagrangian for their couplings is

$$
y\left(\overline{l_{L}} l_{R}\right)_{\underline{\mathbf{1}}} \Phi+\tilde{y}\left(\overline{l_{L}} l_{R}\right)_{\underline{\mathbf{1}}} \tilde{\Phi}+\frac{1}{2} i \tau_{2} f\left(\left(\overline{l_{L}} l_{L}^{c}\right)_{\underline{\mathbf{1}}} \Delta_{L}+\left(\overline{l_{R}^{c}} l_{R}\right)_{\underline{\mathbf{1}}} \Delta_{R}\right)+h . c .
$$

in which the tensor product notations and properties of $A_{4}$ can be found in Appendix A. Then the above assumptions $\mathrm{Eq}(15)$ as well as the lepton mass matrix $M_{e}=\tilde{y} v I$ can be achieved automatically, and they preserve the form of Higgs potential Eq(5) since the Higgs fields now are singlets of $A_{4}$.

In order to obtain non-trivial mixing, we need to introduce another scalar $\Sigma \sim \underline{\mathbf{3}}$ of $A_{4}$ to generate off-diagonal elements and assign the gauge group representation $\Sigma \sim(2,2,0)$ to it. The extra Higgs potential involving $\Sigma$ and the couplings between $\Sigma$ and $\Phi, \Delta_{L(R)}$ are list in the Appendix B. The extra terms that contribute to the $\operatorname{Eq}(7)$ have no effect on the relation $\mathrm{Eq}(9)$, so the results in the previous sections are still valid.

Now the invariant Lagrangian of couplings between leptons and $\Sigma$ is written as

$$
h\left(\bar{l}_{L} l_{R}\right)_{\underline{\mathbf{3}}_{s}} \cdot \Sigma
$$


in which the subscript $\underline{\mathbf{3}}_{s}$ denotes the three dimensional symmetric tensor product as shown in Appendix A. Expanding it into matrix in flavor basis we obtain the extra contributions

$$
\left(\begin{array}{ccc}
0 & h v_{\Sigma_{3}} & h v_{\Sigma_{2}} \\
h v_{\Sigma_{3}} & 0 & h v_{\Sigma_{1}} \\
h v_{\Sigma_{2}} & h v_{\Sigma_{1}} & 0
\end{array}\right)
$$

where $v_{\Sigma_{i}}=\left\langle\Sigma_{i}\right\rangle$. In the assumption of $v_{\Sigma_{1}}=v_{\Sigma_{3}}=0$ and $h v_{\Sigma_{2}}=\delta \neq 0$, the matrix $M_{e}$ and $M_{D}$ have similar forms

$$
M_{e}\left(M_{D}\right)=\tilde{y}(y) v I+\left(\begin{array}{lll}
0 & 0 & \delta \\
0 & 0 & 0 \\
\delta & 0 & 0
\end{array}\right) .
$$

Now, a deviation of $M_{e}$ from $M_{D}$ is needed by perturbations, in general the vanished elements will have non-zero values $\epsilon$, and $\delta$ is perturbed to $\delta^{\prime}$ and $\delta^{\prime \prime}$,

$$
M_{e}=\left(\begin{array}{ccc}
\tilde{y} v & \epsilon_{12} & \delta^{\prime \prime} \\
\epsilon_{21} & \tilde{y} v & \epsilon_{23} \\
\delta^{\prime} & \epsilon_{32} & \tilde{y} v
\end{array}\right)
$$

We assume that $\epsilon_{21}, \epsilon_{32} \simeq \delta^{\prime \prime}$ and $\epsilon_{12}, \epsilon_{23} \simeq \delta^{\prime}$, then we get the mass matrix of charged leptons that can be diagonalized by the unitary matrix

$$
V_{e}=\frac{1}{\sqrt{3}}\left(\begin{array}{ccc}
1 & 1 & 1 \\
1 & \omega & \omega^{2} \\
1 & \omega^{2} & \omega
\end{array}\right)
$$

in which $\omega=e^{\frac{2 \pi i}{3}}$, i.e. $V_{e}^{\dagger} M_{e} V_{e}=\operatorname{diag}\left(m_{e}, m_{\nu}, m_{\tau}\right)$, where

$$
\begin{aligned}
& m_{e}=\tilde{y} v+\delta^{\prime}+\delta^{\prime \prime} \\
& m_{\mu}=\tilde{y} v+\left(\omega \delta^{\prime}+\omega^{2} \delta^{\prime \prime}\right), \\
& m_{\tau}=\tilde{y} v+\left(\omega^{2} \delta^{\prime}+\omega \delta^{\prime \prime}\right) .
\end{aligned}
$$

Under the condition of cancellation relation $\frac{\gamma}{\kappa}=\frac{y^{2}}{f^{2}}$ and non-diagonalized $M_{D} \mathrm{Eq}(20)$, a non-zero neutrino mass matrix now becomes

$$
M_{\nu}=\Delta m I-\frac{2 f v_{L} \delta}{y v}\left(\begin{array}{ccc}
\frac{\delta}{2 y v} & 0 & 1 \\
0 & 0 & 0 \\
1 & 0 & \frac{\delta}{2 y v}
\end{array}\right),
$$

where $\Delta m I$ is a perturbation. $M_{\nu}$ can be diagonalized by the unitary matrix

$$
V_{\nu}=\frac{1}{\sqrt{2}}\left(\begin{array}{ccc}
1 & 0 & -1 \\
0 & \sqrt{2} & 0 \\
1 & 0 & 1
\end{array}\right)
$$

we get 


$$
M_{\nu}^{\text {diag }}=V_{\nu}^{T} M_{\nu} V_{\nu}=\operatorname{diag}\left(\Delta m-\frac{2 f v_{L} \delta}{y v}\left(1+\frac{\delta}{2 y v}\right), \Delta m, \Delta m+\frac{2 f v_{L} \delta}{y v}\left(1-\frac{\delta}{2 y v}\right)\right) .
$$

The MNS matrix [12] is then obtained as

$$
U_{M N S}=V_{e}^{\dagger} V_{\nu}=\left(\begin{array}{ccc}
\frac{2}{\sqrt{6}} & \frac{1}{\sqrt{3}} & 0 \\
-\frac{\omega}{\sqrt{6}} & \frac{\omega}{\sqrt{3}} & -\frac{e^{i \pi / 6}}{\sqrt{2}} \\
-\frac{\omega^{2}}{\sqrt{6}} & \frac{\omega^{2}}{\sqrt{3}} & \frac{e^{-i \pi / 6}}{\sqrt{2}}
\end{array}\right),
$$

which is the tribimaximal mixing matrix up to a phase and hence fits the neutrino oscillation data well.

\section{CONCLUSIONS}

The Type I, II and hybrid (I+II) seesaw mechanisms can be deduced from the LRSM, and classified by the ranges of the parameter $\frac{\gamma}{\kappa}$, which represents the ratio of Higgs particle

self-couplings. Assuming that the Yukawa coupling $y, f$ are of order $O(1)$, then $\frac{\gamma}{\kappa} \ll\left(\frac{y}{f}\right)^{2}$ responds to Type-I seesaw, $\frac{\gamma}{\kappa} \gg\left(\frac{y}{f}\right)^{2}$ to Type-II seesaw and $\frac{\gamma}{\kappa} \simeq\left(\frac{y}{f}\right)^{2}$ to the comparable or nearly cancellation Type-(I+II) seesaw. In the limit of weak couplings in Higgs potential, we find that the parameter region $\frac{\gamma}{\kappa} \simeq\left(\frac{y}{f}\right)^{2} \simeq O(1)$ is more stable against the radiative correction with respect to other regions, hence the nearly cancellation Type-(I+II) is more natural than other types of seesaw in the LRSM.

In the framework of nearly cancellation Type-(I+II) seesaw, the small neutrino masses arise from the cancellation between the contribution of the Type-I and Type-II. In this scenario, the RHN masses can be of order of $\mathrm{O}(10) \mathrm{TeV}$ and be reachable in future colliders. We give a realization of this kind of cancellation scenario by introducing an extra $A_{4}$ flavor symmetry to govern the textures of Yukawa coupling matrices. A realistic model that gives non-zero neutrino masses, charged lepton masses and lepton tribimaximal mixing is also implemented via introducing perturbations to the textures.

\section{ACKNOWLEDGMENTS}

This work was supported in part by the Natural Science Foundation of China under grant No.90203002. 


\section{APPENDIX A: BASIC PROPERTIES OF $A_{4}$}

The $A_{4}$ group has a real three dimensional irreducible representation $\underline{3}$, and three inequivalent one dimensional representation $\underline{\mathbf{1}}, \underline{\mathbf{1}}^{\prime}, \underline{\mathbf{1}}^{\prime \prime}$, in which $\underline{\mathbf{1}}$ stands for the trivial representaion, and $\underline{\mathbf{1}}^{\prime}$ and $\underline{\mathbf{1}}^{\prime \prime}$ are the non-trivial ones and complex conjugates to each other.

The multiplication rules of their non-trivial tensor products are given as

$$
\underline{\mathbf{3}} \otimes \underline{\mathbf{3}}=\underline{\mathbf{3}}_{s} \oplus \underline{\mathbf{3}}_{a} \oplus \underline{\mathbf{1}} \oplus \underline{\mathbf{1}}^{\prime} \oplus \underline{\mathbf{1}}^{\prime \prime} \quad \text { and } \quad \underline{\mathbf{1}}^{\prime} \otimes \underline{\mathbf{1}}^{\prime}=\underline{\mathbf{1}}^{\prime \prime},
$$

in which the subscript $s(a)$ stands for the symmetric (asymmetric) products. If we set $\psi_{i}, \phi_{i} \sim \underline{3}$, then

$$
\begin{aligned}
(\underline{\mathbf{3}} \otimes \underline{\mathbf{3}})_{\underline{\mathbf{1}}} & =\psi_{1} \phi_{1}+\psi_{2} \phi_{2}+\psi_{3} \phi_{3}, \\
(\underline{\mathbf{3}} \otimes \underline{\mathbf{3}})_{\underline{\mathbf{1}}^{\prime}} & =\psi_{1} \phi_{1}+\omega \psi_{2} \phi_{2}+\omega^{2} \psi_{3} \phi_{3}, \\
(\underline{\mathbf{3}} \otimes \underline{\mathbf{3}})_{\underline{1}^{\prime \prime}} & =\psi_{1} \phi_{1}+\omega^{2} \psi_{2} \phi_{2}+\omega \psi_{3} \phi_{3}, \\
(\underline{\mathbf{3}} \otimes \underline{\mathbf{3}})_{\underline{\mathbf{3}}_{s}} & =\left(\psi_{2} \phi_{3}+\psi_{3} \phi_{2}, \psi_{3} \phi_{1}+\psi_{1} \phi_{3}, \psi_{1} \phi_{2}+\psi_{2} \phi_{1}\right), \\
\left(\underline{\mathbf{3}} \otimes \underline{\mathbf{3}}_{)_{a}}\right. & =\left(\psi_{2} \phi_{3}-\psi_{3} \phi_{2}, \psi_{3} \phi_{1}-\psi_{1} \phi_{3}, \psi_{1} \phi_{2}-\psi_{2} \phi_{1}\right),
\end{aligned}
$$

with $\omega=e^{\frac{2 \pi i}{3}}$.

\section{APPENDIX B: HIGGS POTENTIAL}

In addition to the $A_{4}$ singlet Higgs fields $\Phi$ and $\Delta_{L(R)}$, we have introduced another scalar $\Sigma \sim(2,2,0)(\underline{\mathbf{3}})$ under the group $G_{L R} \otimes A_{4}$, so the extra Higgs potential involving $\Sigma$ should be added. The potential involving $\Phi$ and $\Delta_{L(R)}$ preserves its form $\operatorname{Eq}(5)$ since they are trivial representation of $A_{4}$, i.e. $\Phi, \Delta_{L(R)} \sim \underline{\mathbf{1}}$, we will not write them here again. According to the representation assignment of $\Sigma$, the invariant potential can be written as

$$
\begin{aligned}
V(\Sigma) & =\mu_{\Sigma}^{2}\left(\Sigma^{\dagger} \Sigma\right)_{\underline{\mathbf{1}}}+\lambda_{1}^{\Sigma}\left(\Sigma^{\dagger} \Sigma\right)_{\underline{\mathbf{1}}}\left(\Sigma^{\dagger} \Sigma\right)_{\mathbf{1}}+\lambda_{2}^{\Sigma}\left(\Sigma^{\dagger} \Sigma\right)_{\underline{\mathbf{1}}^{\prime}}\left(\Sigma^{\dagger} \Sigma\right)_{\underline{\mathbf{1}}^{\prime \prime}} \\
& +\lambda_{3}^{\Sigma}\left(\Sigma^{\dagger} \Sigma\right)_{\underline{\mathbf{3}}_{s}}\left(\Sigma^{\dagger} \Sigma\right)_{\underline{\mathbf{3}}_{s}}+\lambda_{4}^{\Sigma}\left(\Sigma^{\dagger} \Sigma\right)_{\underline{\mathbf{3}}_{a}}\left(\Sigma^{\dagger} \Sigma\right)_{\underline{\mathbf{3}}_{a}} \\
& +i \lambda_{5}^{\Sigma}\left(\Sigma^{\dagger} \Sigma\right)_{\underline{\mathbf{3}}_{s}}\left(\Sigma^{\dagger} \Sigma\right)_{\underline{\mathbf{3}}_{a}}, \\
V(\Phi, \Sigma) & =\lambda_{1}^{\Phi \Sigma}\left(\Sigma^{\dagger} \Sigma\right)_{\underline{\mathbf{1}}}\left(\Phi^{\dagger} \Phi\right)_{\underline{\mathbf{1}}}+\lambda_{2}^{\Phi \Sigma}\left(\Sigma^{\dagger} \Phi\right)_{\underline{\mathbf{3}}}\left(\Phi^{\dagger} \Sigma\right)_{\underline{\mathbf{3}}} \\
& +\lambda_{3}^{\Phi \Sigma}\left(\Sigma^{\dagger} \Phi\right)_{\underline{\mathbf{3}}}\left(\Sigma^{\dagger} \Phi\right)_{\underline{\mathbf{3}}}+h . c ., \\
V\left(\Delta_{L}, \Delta_{R}, \Sigma\right) & =\lambda_{1}^{\Delta \Sigma}\left[\operatorname{tr}\left(\Delta_{L}^{\dagger} \Delta_{L}\right)_{\underline{\mathbf{1}}}+\operatorname{tr}\left(\Delta_{R}^{\dagger} \Delta_{R}\right)_{\underline{\mathbf{1}}}\right]\left(\Sigma^{\dagger} \Sigma\right)_{\underline{\mathbf{1}}} \\
& +\lambda_{2}^{\Delta \Sigma} \Sigma^{\dagger}\left(\left[\Delta_{L}, \Delta_{L}^{\dagger}\right]_{\underline{\mathbf{1}}}+\left[\Delta_{R}, \Delta_{R}^{\dagger}\right]_{\underline{\mathbf{1}}}\right) \Sigma_{\underline{\mathbf{3}}} .
\end{aligned}
$$

There is no renormalizable term simultaneously involving $\Phi, \Delta_{L(R)}$ and $\Sigma$,

$$
V\left(\Phi, \Delta_{L}, \Delta_{R}, \Sigma\right)=0
$$

So the total Higgs potential is given by

$$
V=V\left(\Phi, \Delta_{L}, \Delta_{R}\right)+V(\Sigma)+V(\Phi, \Sigma)+V\left(\Delta_{L}, \Delta_{R}, \Sigma\right) .
$$




\section{REFERENCES}

[1] SNO Collaboration, Q.R. Ahmad et al., Phys. Rev. Lett. 89, 011301 (2002). Atmospheric experiment, C.K. Jung et al., Ann. Rev. Nucl. Part. Sci. 51, 451 (2001). KamLAND Collaboration, K. Eguchi et al., Phys. Rev. Lett. 90, 021802 (2003).

K2K Collaboration, M.H. Ahn et al., Phys. Rev. Lett. 90, 041801 (2003).

[2] P. Minkowski, Phys. Lett. B 67, 421 (1977); T. Yanagida, in Proceedings of the Workshop on Unified Theory and the Baryon Number of the Universe, edited by O. Sawada and A. Sugamoto (KEK, Tsukuba, 1979), p. 95; M. Gell-Mann, P. Ramond, and R. Slansky, in Supergravity, edited by F. van Nieuwenhuizen and D. Freedman (North Holland, Amsterdam, 1979), p. 315; S.L. Glashow, in Quarks and Leptons, edited by M. Lévy et al. (Plenum, New York, 1980), p. 707; R.N. Mohapatra and G. Senjanovic, Phys. Rev. Lett. 44, 912 (1980).

[3] J.Schechter, J.W.F.Valle, Phys.Rev.D 22,2227 (1980); T.P.Cheng and L.F.Li,Phys.Rev.D 22, 2860 (1980); M.Magg and C.Wetterich, Phys.Lett.B 94, 61 (1980); R.N.Mohapatra and G.Senjanovic, Phy.Rev.D 23, 165 (1981).

[4] W.Chao, S.Luo, Z.Z.Xing, and S.Zhou, Phys.Rev.D 77, 016001 (2008).

[5] F.del Aguila, J.A.Aguilar-Saavedra, A.Martinez de la Ossa, and D.Meloni,Phys.Lett.B 613 (2005), 170, hep-ph/0502189; T.Han and B.Zhang, Phys.Rev.Lett. 97 (2006), 171804, hep-ph/0604064; F.del Aguila, J.A.Aguilar-Saavedra, and R.Pittau, J.Phys.Conf.Ser.53 (2006),506, hep-ph/0606198; S.Bray, J.S.Lee, and A.Pilaftsis, hepph/0702294. F.del Aguila, J.A.Aguilar-Saavedra, and R.Pittau, hep-ph/0703261.

[6] J.C. Pati and A. Salam, Phys. Rev. D 10, 275 (1974); R.N. Mohapatra and J.C. Pati, Phys. Rev. D 11, 566 (1975); Phys. Rev. D 11, 2558 (1975); G. Senjanovic and R.N. Mohapatra, Phys. Rev. D 12, 1502 (1975).

[7] P.F. Harrison, D.H. Perkins, and W.G. Scott, Phys. Lett. B 530, 167 (2002); Z.Z. Xing, Phys. Lett. B 533, 85 (2002); P.F. Harrison and W.G. Scott, Phys. Lett. B 535, 163 (2002); X.G. He and A. Zee, Phys. Lett. B 560, 87 (2003).

[8] E. Ma and G. Rajasekaran, Phys. Rev. D 64, 113012 (2001); K.S. Babu, E. Ma, and J.W.F. Valle, Phys. Lett. B 552, 207 (2003); E. Ma, Phys. Rev. D 70, 031001 (2004); ibid 72, 037301 (2005); Mod. Phys. Lett. A 20, 2601 (2005); G. Altarelli and F. Feruglio, Nucl. Phys. B 720, 64 (2005); ibid 741, 215 (2006); K.S. Babu and X.G. He, hepph/0507217; A. Zee, Phys. Lett. B 630, 58 (2006); M. Hirsch, A.S. Joshipura, S. Kaneko, and J.W.F. Valle, hep-ph/0703046.

[9] W.M.Yao et al., J.Phys.G 33,1 (2006)

[10] R.N.Mohapatra and G.Senjanov, Phys.Rev.D 23, 165 (1981); J.F.Gunion, J.Grifols and A.Mendez, Phys.Rev.D 40, 1546 (1989).

[11] E.K.Akhmedov and M.Frigerio, Phys.Rev.Lett.96, 061802 (2006), hep-ph/0509299

[12] Z.Maki, M.Nakagawa and S.Sakata, Prog.Theor.Phys. bf 28 (1962) 870. 